\title{
Recruitment of participants through community pharmacies for a pharmacogenetic study of antihypertensive drug treatment
}

\author{
Diane B. M. A. van Wieren-de Wijer • Anke-Hilse Maitland-van der Zee • \\ Anthonius de Boer · Bruno H. Ch. Stricker · Abraham A. Kroon • \\ Peter W. de Leeuw • O. Bozkurt • Olaf H. Klungel
}

Received: 4 June 2008/Accepted: 2 November 2008/Published online: 30 November 2008

(c) The Author(s) 2008. This article is published with open access at Springerlink.com

\begin{abstract}
Objective To describe the design, recruitment and baseline characteristics of participants in a community pharmacy based pharmacogenetic study of antihypertensive drug treatment. Setting: Participants enrolled from the population-based Pharmaco-Morbidity Record Linkage System. Method We designed a nested case-control study in which we will assess whether specific genetic polymorphisms modify the effect of antihypertensive drugs on the risk of myocardial infarction. In this study, cases (myocardial infarction) and controls were recruited through community pharmacies that participate in PHARMO. The PHARMO database comprises drug dispensing histories of about 2,000,000 subjects from a representative sample of Dutch community pharmacies linked to the national registrations of hospital discharges. Results In total we selected 31010 patients (2777 cases and 28233 controls)
\end{abstract}

D. B. M. A. van Wieren-de Wijer - A.-H. Maitland-van der Zee · A. de Boer · O. Bozkurt · O. H. Klungel ( $\square)$

Department of Pharmacoepidemiology \& Pharmacotherapy, Utrecht Institute for Pharmaceutical Sciences (UIPS), University of Utrecht, P.O. Box 80082, 3508 TB Utrecht, The Netherlands e-mail: o.h.klungel@uu.nl

D. B. M. A. van Wieren-de Wijer - B. H. Ch. Stricker Department of Epidemiology \& Biostatistics, Pharmaco-epidemiology Unit, Erasmus Medical Centre, Rotterdam, The Netherlands

A. A. Kroon

Department of Internal Medicine Cardiovascular Research Institute Maastricht (CARIM), University of Maastricht,

Maastricht, The Netherlands

P. W. de Leeuw

Department of Pharmacology and Toxicology, Cardiovascular

Research Institute Maastricht (CARIM), University of

Maastricht, Maastricht, The Netherlands from the PHARMO database, of whom 15973 (1871 cases, 14102 controls) were approached through their community pharmacy. Overall response rate was $36.3 \% \quad(n=5791$, 794 cases, 4997 controls), whereas $32.1 \%$ ( $n=5126,701$ cases, 4425 controls) gave informed consent to genotype their DNA. As expected, several cardiovascular risk factors such as smoking, body mass index, hypercholesterolemia, and diabetes mellitus were more common in cases than in controls. Conclusion Furthermore, cases more often used beta-blockers and calcium-antagonists, whereas controls more often used thiazide diuretics, ACE-inhibitors, and angiotensin-II receptor blockers. We have demonstrated that it is feasible to select patients from a coded database for a pharmacogenetic study and to approach them through community pharmacies, achieving reasonable response rates and without violating privacy rules.

Keywords Community pharmacy - Hypertension · Patient recruitment · Pharmacogenetics - Netherlands

\section{Impact of findings on practice}

- Pharmacogenetic research is important for clinical practice because it will facilitate personalized medicine in the (near) future.

- For antihypertensive drugs that are commonly used conducting a clinical trial will lead to practical constraints, therefore it is very important to perform observational pharmacogenetic research.

- This study shows that it is feasible to perform patient recruitment for this observational pharmacogenetic research in community pharmacies in The Netherlands. 
- Pharmacists should play an important role in introducing pharmacogenetics into clinical practice; both in the research and in the implementation phase.

\section{Introduction}

High blood pressure is a common and major risk factor for cardiovascular disease, affecting approximately $25 \%$ of the industrialized countries [1]. Despite the availability of a variety of effective antihypertensive drugs, inadequate control of blood pressure is common in hypertensive patients, and responsible for a large proportion of the burden of stroke and myocardial infarction (MI) in the population [2,3]. Although there are some weak predictors of response to antihypertensive drugs, individualization of treatment is mostly done empirically [4]. A better knowledge of the mechanisms underlying individual variation in the effectiveness of drugs may improve this situation. Identification of genes which modify the response to antihypertensive drugs provides the opportunity to optimize safety and effectiveness of the currently available antihypertensive drugs, and to design new drugs.

Studies of drug-gene interactions may be conducted from a pharmacokinetic and a pharmacodynamic perspective $[5,6]$. From a pharmacodynamic perspective, several small studies have evaluated the influence of polymorphisms in renin-angiotensin system genes (angiotensin converting enzyme (ACE), angiotensinogen (AGT), angiotensin II type 1 receptor (AT1R)), the $\alpha$-adducin gene, and the $\beta$-adrenoceptor ( $\beta \mathrm{AR}$ )-G protein system genes on the response to antihypertensive drugs [7-11]. These studies focused on blood pressure, arterial stiffness, or regression of left ventricular hypertrophy as outcomes. Although some suggested interactions between certain genetic polymorphisms and antihypertensive therapy, results were often inconsistent due to small sample sizes, confounding factors, differences between populations, focus on one single gene and failure to account for genegene interactions [12]. These treatment-gene interactions have not been evaluated extensively in large populationbased studies and the effects on clinically important outcomes such as myocardial infarction are largely unknown. Some studies in which genetic variation as determinant of antihypertensive drug response was determined gave inconsistent results [13-16]. Therefore it is important that large population-based studies are performed to enable comparing and possibly pooling of results and eventually finding true drug-gene interactions.

We designed a nested case-control study in which we will assess whether specific genetic polymorphisms modify the effect of antihypertensive drugs on the risk of myocardial infarction. In our study the objective is to assess interactions between antihypertensive drugs (notably diuretics, ACE-inhibitors, $\beta$-blockers and calcium antagonists) and genetic polymorphisms (e.g. AGT, $\alpha$-adducin, Gp- $\beta_{3}$, ACE, AT1R, G- $\alpha$ protein, $\beta-2$ and $\beta-1$ adrenergic receptors, epithelial sodium channel (EnaC), cytochrome P-450 enzymes (CYP2D6, CYP2C9, CYP3A4), and P-glycoprotein) with respect to cardiovascular outcomes. In this study, subjects were recruited through community pharmacies. This paper describes the design and implementation of the study, and shows the baseline characteristics of subjects who participate in the study.

\section{Methods}

Design and setting

A nested case-control design was used to assess whether specific genetic polymorphisms modify the effect of antihypertensive drugs on the risk of myocardial infarction.

Participants were enrolled from the population-based Pharmaco-Morbidity Record Linkage System (PHARMO). PHARMO links drug dispensing histories from a representative sample of Dutch community pharmacies to the national registrations of hospital discharges (LMR) from 1985 onwards. Currently, the base population of PHARMO covers about 2,000,000 community-dwelling inhabitants of several population-defined areas in The Netherlands, a sample comparable to the general Dutch population.

Approval for this study was obtained from the Medical Ethics Committee of the University Medical Center Utrecht, The Netherlands.

\section{Case and control definition}

In the PHARMO database subjects who used antihypertensive drugs (low-ceiling diuretics, $\beta$-blockers, ACEinhibitors, calcium antagonists, angiotensin-II type 1 receptor blockers (ARB), miscellaneous antihypertensives and combinations of antihypertensives) were selected. From this cohort, subjects hospitalised for MI (ICD-9 code 410) were included as cases if they had at least one prescription for antihypertensive drugs in the 3 months prior to their first MI hospitalisation and were registered in PHARMO for at least 1 year. The index date was defined as the date of admission for the first myocardial infarction. Patients were excluded if they were $<18$ years of age, if the last prescription was not more than 100 days before index date (90 days plus 10 days to account for irregularity of refills), if they had had a previous MI, or if the date of birth and sex filled in the questionnaire did not match the data in the PHARMO database. Initially we randomly selected six control subjects for each case from the subset of 
antihypertensive drug users, assuming a 50\% response rate. All non-responders were contacted by telephone. However, because the response rate was lower than expected and because the effort to include a sufficient number of controls per case was substantial, the number of controls we selected for each case was later increased to 12 and non-responsive controls were not contacted anymore. Controls met the same eligibility criteria as the cases, but did not have an MI. They were matched to the cases on age ( \pm 1 year), sex and area of residence and were assigned the same index date as the case to whom they had been matched.

\section{Patient recruitment}

From the PHARMO institute a list with patient identifying numbers which were linked to patient research numbers was available. Subjects were recruited through community pharmacies, which participate in PHARMO. From the participating pharmacy the subjects received a letter in which the purpose of the study was explained. They were asked to return an informed consent form and a questionnaire to the PHARMO institute. The informed consent form contains name, birth date and patient identifying number and is stored separately at the PHARMO institute. The questionnaire contains the patient research number. After the participant had consented to participate in the study (s)he was sent a package with three tubes with the patient research number and three cotton swabs for a buccal swab procedure. All participants were explicitly asked to consent for the collection, storage and genotyping of the buccal swab material. All patients were notified that we used information from general practitioners through anonymous linkage with the pharmacy data.

\section{Ascertainment of exposure to antihypertensive drugs}

Coded pharmacy records were used to ascertain exposure to antihypertensive drugs. In PHARMO, complete pharmacy records are available as of 1991. Pharmacy records provide details on day of delivery, daily dose, and durations of therapy. We distinguished major antihypertensive drug classes such as low-ceiling diuretics, $\beta$-blockers, ACE-inhibitors, calcium antagonists, angiotensin-II type 1 receptor blockers (ARB), miscellaneous antihypertensives and combinations of antihypertensives. Compliance was judged from the regularity of refills.

\section{Assessment of potential confounding factors and effect-modifiers}

In our study we have collected information on smoking, hypertension, hypercholesterolemia, diabetes mellitus, use of alcohol, diets, history of cardiovascular diseases, family history of cardiovascular diseases, weight and height through self-administered questionnaires. For a part of the population information from automated general practice and laboratory registrations is available. For all participants, information about risk factors was assessed before the index date.

\section{Buccal cell collection and DNA extraction}

Individuals who agreed to participate in the study were asked to supply a sample of buccal cells, collected by the participants themselves. They received one page of collection instructions, three cotton swabs, three $15 \mathrm{ml}$ tubes containing $2 \mathrm{ml}$ buffer (1880 $\mu \mathrm{l}$ STE $(100 \mathrm{mM} \mathrm{NaCl}$, $10 \mathrm{mM}$ EDTA, $10 \mathrm{mM}$ Tris), $100 \mu \mathrm{l} 10 \%$ SDS and $20 \mu \mathrm{l}$ of $10 \mathrm{mg} / \mathrm{ml}$ Proteinase $\mathrm{K}$ ).

\section{Genotyping}

Genotypes of the $\alpha$-adducin, ACE, angiotensinogen, angiotensin II type 1 receptor, eNOS and G-protein- $\beta_{3}$, polymorphisms were assessed using a multiplex single base extension (SBE) method. Multiplex SBE was performed using $\mathrm{SNaPshot}^{\mathrm{TM}}$ as described by the manufacturer (Applied Biosystems). This method was described earlier but adapted to this new set of polymorphisms. Laboratory personnel were blinded both to case-control status and to antihypertensive drug-therapy. Briefly, this assay uses pooled PCR primer pairs to co-amplify 27 targets from genomic DNA in two reactions. Amplified fragments within each PCR products pool are then detected colorimetrically with sequence-specific oligonucleotide probes immobilized in a linear array on nylon membranes [17]. Table 1 shows an overview of the polymorphisms genotyped in the PHARMO-study linked to the antihypertensive drug class that might be influenced by this polymorphisms. This selection was made based on literature search and the knowledge of the action mechanism of the drugs.

\section{Analysis}

Conditional logistic regression analysis will be used to study the association between antihypertensive drug use and the incidence of myocardial infarction and to adjust for potential confounding. All analyses will be stratified by the genetic polymorphisms to study effect modification. Analyses will also be stratified by antihypertensive drug class. Stratified analysis will also be used to account for gene-gene interactions. Interaction terms (drug $\times$ genotype) will be included in the logistic regression model to calculate synergy indices (SI). We will test genotypeeffect, dominant and recessive models, and allele-effect associations. 
Table 1 Summary of the polymorphisms genotyped in the PHARMO study that might influence antihypertensive drug therapy by drug class

\begin{tabular}{|c|c|c|c|}
\hline Drug & $\begin{array}{l}\text { Polymorphism } \\
\text { (nucleotide substitution) }\end{array}$ & Amino Acid substitution (3-coded; 1-coded) & $\begin{array}{l}\text { dbSNP } \\
\text { rs number }\end{array}$ \\
\hline \multirow[t]{7}{*}{ Thiazide diuretics } & ADD1 G460T & Glycine-Trytophane (gly-trp; G-W) & 4961 \\
\hline & AGT C235T & Methionine-Threonine (met-thr; M-T) & 699 \\
\hline & ACE G4656C & Non-coding & 4341 \\
\hline & ACE T3892C & Non-coding & 1800764 \\
\hline & AGTR1 A1166C & Non-coding & 5186 \\
\hline & GNB3 C825T & No substitution (Serine-Serine; ser; S) & 5443 \\
\hline & NOS3 G298T & Aspartic Acid-Glutamic Acid (Asp-Glu; D-E) & 1799983 \\
\hline \multirow[t]{5}{*}{ Beta-blocker } & AGT C235T & Methionine-Threonine (met-thr; M-T) & 699 \\
\hline & ACE G4656C & Non-coding & 4341 \\
\hline & ACE T3892C & Non-coding & 1800764 \\
\hline & AGTR1 A1166C & Non-coding & 5186 \\
\hline & GNB3 C825T & No substitution (Serine-Serine; ser; S) & 5443 \\
\hline \multirow[t]{4}{*}{ ACE-inhibitor } & AGT C235T & Methionine-Threonine (met-thr; M-T) & 699 \\
\hline & ACE G4656C & Non-coding & 4341 \\
\hline & ACE T3892C & Non-coding & 1800764 \\
\hline & AGTR1 A1166C & Non-coding & 5186 \\
\hline \multirow[t]{4}{*}{ AT1R antagonist } & AGT C235T & Methionine-Threonine (met-thr; $\mathrm{M}-\mathrm{T}$ ) & 699 \\
\hline & ACE G4656C & Non-coding & 4341 \\
\hline & ACE T3892C & Non-coding & 1800764 \\
\hline & AGTR1 A1166C & Non-coding & 5186 \\
\hline \multirow[t]{5}{*}{ Calcium channel blocker } & AGT C235T & Methionine-Threonine (met-thr; $\mathrm{M}-\mathrm{T}$ ) & 699 \\
\hline & ACE G4656C & Non-coding & 4341 \\
\hline & ACE T3892C & Non-coding & 1800764 \\
\hline & AGTR1 A1166C & Non-coding & 5186 \\
\hline & NOS3 G298T & Aspartic Acid-Glutamic Acid (Asp-Glu; D-E) & 1799983 \\
\hline
\end{tabular}

\section{Results}

In total 31,010 patients (2,777 cases and 28,233 controls) were selected from the PHARMO-database, of whom 15,973 were approached by their pharmacies. The other 15,037 patients could not be approached for different reasons (for example they died or moved or for controls the case to whom they were matched did not participate). In total 5,791 patients responded by filling in the questionnaire, which gave an overall response rate of $36.3 \%$, and $5,126(31.7 \%)$ gave informed consent to genotype their DNA. Baseline characteristics from the 794 cases and 4,997 controls are shown in Table 2. As expected the determinants smoking, body mass index, hypercholesterolemia, and diabetes mellitus were different between cases and controls. Furthermore, beta-blockers and calcium-antagonists were used more often by cases than controls, whereas thiazide diuretics, ACE-inhibitors and angiotensin-II blockers were used more often by controls than cases. Ethnicity did not differ between cases and controls and because of the matching neither did age nor did gender.

\section{Discussion}

The recently completed sequencing of the human genome has focused attention on the potential for genetic information to benefit the diagnosis, evaluation and treatment of common diseases. Pharmacogenetics is a research field that pursues the identification and characterization of genes that influence individual responses to drug treatment. The availability of more and more data on the existence of genes and their variability, will give rise to an increase in pharmacogenetic research. In the field of hypertension, an important goal is to ascertain which of the genes influence the effects of antihypertensive drugs on blood pressure, assess the extent and impact of their polymorphisms and distinguish which pathways are valid targets for intervention. Although the public health importance of the results of pharmacogenetic studies may be considerable, reliable information is still limited in this area and needs to be assessed.

We have designed a nested case-control study in which we will assess whether genetic polymorphisms modify the effect of antihypertensive drugs on the risk of MI. In our 
Table 2 Baseline characteristics of the participants

\begin{tabular}{|c|c|c|c|}
\hline & $\begin{array}{l}\text { Cases }(n=794) \\
\text { Number }(\%)\end{array}$ & $\begin{array}{l}\text { Controls (4997) } \\
\text { Number }(\%)\end{array}$ & $P$-value \\
\hline Age in years (SD) & $64.8(10.8)$ & $64.5(10.3)$ & \\
\hline Female & $261(32.9)$ & $1,639(32.8)$ & \\
\hline Consent DNA & $701(88.3)$ & 4,427 (88.6) & \\
\hline DNA available & $631(79.5)$ & $3,952(79.1)$ & \\
\hline \multicolumn{4}{|l|}{ Antihypertensive drugs } \\
\hline Thiazide diuretics & $131(16.8)$ & $1,204(24.5)$ & $<0.0005$ \\
\hline Beta blockers & $362(46.4)$ & $2,344(47.8)$ & 0.48 \\
\hline ACE-inhibitors & $173(22.2)$ & $1,539(31.4)$ & $<0.0005$ \\
\hline Ca-antagonists & $232(29.7)$ & $1,061(21.6)$ & $<0.0005$ \\
\hline AT2-antagonists & $66(8.5)$ & $682(13.9)$ & $<0.0005$ \\
\hline Alpha-blockers & $10(1.3)$ & $69(1.4)$ & 0.78 \\
\hline Combination & $65(8.3)$ & $627(12.8)$ & $<0.0005$ \\
\hline Smoking & $N=720$ & $N=4,597$ & \\
\hline Never & $226(31.4)$ & $1,769(38.5)$ & $<0.0005$ \\
\hline Current & $154(21.4)$ & $695(15.1)$ & \\
\hline Past & $340(47.2)$ & $2,133(46.4)$ & \\
\hline BMI & $N=688$ & $N=4,200$ & \\
\hline$>30 \mathrm{~kg} / \mathrm{m}^{2}$ & $164(23.8)$ & $849(20.2)$ & 0.03 \\
\hline Hypercholesterolemia & $N=757$ & $N=4,810$ & \\
\hline No & $258(34.1)$ & $2,438(50.7)$ & $<0.0005$ \\
\hline Yes, no drug treatment & $306(40.4)$ & $918(19.1)$ & \\
\hline Yes, drug treatment & $193(25.5)$ & $1,454(30.2)$ & \\
\hline Diabetes mellitus & $N=766$ & $N=4,845$ & \\
\hline No & $593(77.4)$ & $3,914(80.8)$ & 0.02 \\
\hline Yes, no drug treatment & $80(10.4)$ & $367(7.6)$ & \\
\hline Yes, drug treatment & $93(12.1)$ & $564(11.6)$ & \\
\hline Physical activity & $N=776$ & $N=4,881$ & \\
\hline$<4 \mathrm{~h}$ & $238(30.7)$ & $1,303(26.7)$ & 0.02 \\
\hline$\geq 4 \mathrm{~h}$ & $538(69.3)$ & $3,578(73.3)$ & \\
\hline Alcohol use & $N=761$ & $N=4790$ & \\
\hline no use & $173(22.7)$ & $966(20.2)$ & 0.02 \\
\hline$<1$ per day & $192(25.3)$ & $1,194(24.9)$ & \\
\hline $1-2$ per day & $41(5.4)$ & $360(7.5)$ & \\
\hline$>2$ per day & $25(3.3)$ & $258(5.4)$ & \\
\hline yes (unknown quantity) & $330(43.4)$ & $2,012(42.0)$ & \\
\hline Family history MI & $N=780$ & $N=4903$ & \\
\hline No & $724(92.8)$ & 4,642 (94.7) & 0.08 \\
\hline Yes $<60$ years & $6(0.8)$ & $20(0.4)$ & \\
\hline Yes $>60$ years & $50(6.4)$ & $241(4.9)$ & \\
\hline Ethnicity & $N=776$ & $N=4,868$ & \\
\hline Caucasian & $759(97.8)$ & $4,746(97.5)$ & 0.75 \\
\hline Other & $17(2.2)$ & $122(2.5)$ & \\
\hline
\end{tabular}

study we have access to data on all different antihypertensive drugs and therefore include different calcium channel blockers, ACE inhibitors, alpha-adrenergic blockers and diuretics. Furthermore, in our study data is also available on the use of $\beta$-blockers.
A limitation of our study is the use of self-reported data. However, in about $40 \%$ of the PHARMO population, computerized general practice and clinical laboratory data are available. This will allow us to conduct several sensitivity analyses and validation studies. Another limitation is 
the use of computerized pharmacy data. The pharmacy records measured prescriptions filled rather than drugs taken. Moreover antihypertensive drug users with fatal MI were not selected for the study because they were not registered in the PHARMO database.

A number of studies have investigated genetic polymorphisms as determinants of cardiovascular response to antihypertensive drug therapy. They used different study designs such as experimental (e.g. randomized clinical trial) and observational (e.g. cohort and case-control) studies. Data from randomized controlled trials constitute the highest order of evidence and remain the standard for comparisons between therapies [18]. However, randomized controlled trials may not always feasible due to practical, financial or ethical reasons. Many clinical trials that are performed now are saving blood specimens, but most earlier trials do not have blood samples available. Observational studies are the main alternatives [19]. Moreover observational studies represent a more accurate accounting of everyday clinical care and estimates of treatment effects are more generalisable to the general population. In observational studies factors that determined whether a patient received a specific drug or not could result in difference between groups in prognostics factors related to the outcome. Specific patient characteristics, clinical judgment and consideration of the best option for the patient will influence the decision of the treating physician to prescribe a specific antihypertensive drug. For instance hypertensive patients with diabetes mellitus are more likely to receive ACE-inhibitors. However in observational pharmacogenetic studies drug-gene interactions are probably not influenced by these problems, as the prescriber is unaware of a patient's genotype. Information from both randomized controlled trials and outcomes databases is necessary to determine appropriate treatment for individual patients.

The unique and new feature about our study is the enrollment of the subjects. All subjects were approached through the community pharmacies which participate in PHARMO. The enforcement of this population based study is therefore relatively easy and the threshold to participate is kept as low as possible.

\section{Conclusion}

This study demonstrates the feasibility of setting up a retrospective study by collecting information through community pharmacies and patients themselves.

The results of this study will help to assess the clinical relevance of antihypertensive drug-gene interactions. Understanding the association between antihypertensive drug-gene interactions and various cardiovascular outcomes may eventually help physicians and other health professionals to tailor antihypertensive drug therapy to the individual patient.

Acknowledgements The authors would like to thank the following for their support during the project implementation: Karin J.A. Lievers at University of Utrecht, P. Schiffers and R.J. Janssen at University of Maastricht, Cornelia M. van Duijn at Erasmus Medical Centre Rotterdam, Bruce M. Psaty at University of Washington and W.M. Monique Verschuren at National Institute of Public Health and the Environment Bilthoven.

Funding This study is funded by grant no. 2001.064 of The Netherlands Heart Foundation. A.H. Maitland-van der Zee is funded by a Veni grant from the Netherlands Organization for Scientific Research (NWO)

Conflicts of Interest The department employing authors BMAW, AHM, OHK, AB receives unrestricted research grants for pharmacoepidemiological research from the private-public funded Top Institute Pharma (includes co-funding from universities, government, and industry-GSK, Organon, Merck) and GSK. The authors AB and OHK received an unrestricted research grant for pharmacoepidemiological research on diabetes mellitus.

The experiments in this study comply with the current laws in The Netherlands. Approval for this study was obtained from the Medical Ethics Committee of the University Medical Center Utrecht, The Netherlands.

Open Access This article is distributed under the terms of the Creative Commons Attribution Noncommercial License which permits any noncommercial use, distribution, and reproduction in any medium, provided the original author(s) and source are credited.

\section{Reference}

1. Mosterd A, D'Agostino RB, Silbershatz H, Sytkowski PA, Kannel WB, Grobbee DE, et al. Trends in the prevalence of hypertension, antihypertensive therapy, and left ventricular hypertrophy from 1950 to 1989 . N Engl J Med. 1999;340:1221-7. doi:10.1056/NEJM199904223401601.

2. Klungel OH, Stricker BH, Paes AH, Seidell JC, Bakker A, Voko $\mathrm{Z}$, et al. Excess stroke among hypertensive men and women attributable to undertreatment of hypertension. Stroke. 1999;30: 1312-8.

3. Kaplan RC, Psaty BM, Heckbert SR, Smith NL, Lemaitre RN. Blood pressure level and incidence of myocardial infarction among patients treated for hypertension. Am J Public Health. 1999;89:1414-7.

4. Weder AB. Selecting the right drug for initial antihypertensive therapy. Curr Hypertens Rep. 2000;2:13-5. doi:10.1007/s11906000-0052-9.

5. Evans WE, Relling MV. Pharmacogenomics: translating functional genomics into rational therapeutics. Science. 1999;286:487-91. doi:10.1126/science.286.5439.487.

6. Maitland-van der Zee AH, de Boer A, Leufkens HG. The interface between pharmacoepidemiology and pharmacogenetics. Eur J Pharmacol. 2000;410:121-30. doi:10.1016/S0014-2999(00) 00810-4.

7. Cusi D, Barlassina C, Azzani T, Casari G, Citterio L, Devoto M, et al. Polymorphisms of alpha-adducin and salt sensitivity in patients with essential hypertension. Lancet. 1997;349:1353-7. doi:10.1016/S0140-6736(97)01029-5. 
8. Hingorani AD, Jia H, Stevens PA, Hopper R, Dickerson JE, Brown MJ. Renin-angiotensin system gene polymorphisms influence blood pressure and the response to angiotensin converting enzyme inhibition. J Hypertens. 1995;13:1602-9.

9. Benetos A, Cambien F, Gautier S, Ricard S, Safar M, Laurent S, et al. Influence of the angiotensin II type 1 receptor gene polymorphism on the effects of perindopril and nitrendipine on arterial stiffness in hypertensive individuals. Hypertension. 1996;28:1081-4.

10. Jia H, Hingorani AD, Sharma $P$, Hopper $R$, Dickerson $C$, Trutwein D, et al. Association of the G(s)alpha gene with essential hypertension and response to beta-blockade. Hypertension. 1999;34:8-14.

11. Kohno M, Yokokawa K, Minami M, Kano H, Yasunari K, Hanehira T, et al. Association between angiotensin-converting enzyme gene polymorphisms and regression of left ventricular hypertrophy in patients treated with angiotensin-converting enzyme inhibitors. Am J Med. 1999;106:544-9. doi:10.1016/ S0002-9343(99)00067-4.

12. Williams SM, Addy JH, Phillips JAIII, Dai M, Kpodonu J, Afful $\mathrm{J}$, et al. Combinations of variations in multiple genes are associated with hypertension. Hypertension. 2000;36:2-6.

13. Arnett DK, Davis BR, Ford CE, Boerwinkle E, LeiendeckerFoster C, Miller MB, et al. Pharmacogenetic association of the angiotensin-converting enzyme insertion/deletion polymorphism on blood pressure and cardiovascular risk in relation to antihypertensive treatment: the Genetics of Hypertension-Associated Treatment (GenHAT) study. Circulation. 2005;111:3374-83. doi: 10.1161/CIRCULATIONAHA.104.504639.
14. Davis BR, Arnett DK, Boerwinkle E, Ford CE, LeiendeckerFoster C, Miller MB, et al. Antihypertensive therapy, the alphaadducin polymorphism, and cardiovascular disease in high-risk hypertensive persons: the Genetics of Hypertension-Associated Treatment Study. Pharmacogenomics J. 2007;7:112-22. doi: 10.1038/sj.tpj.6500395.

15. Psaty BM, Smith NL, Heckbert SR, Vos HL, Lemaitre RN, Reiner AP, et al. Diuretic therapy, the alpha-adducin gene variant, and the risk of myocardial infarction or stroke in persons with treated hypertension. JAMA. 2002;287:1680-9. doi:10.1001/ jama.287.13.1680.

16. Bis JC, Smith NL, Psaty BM, Heckbert SR, Edwards KL, Lemaitre RN, et al. Angiotensinogen Met235Thr polymorphism, angiotensin-converting enzyme inhibitor therapy, and the risk of nonfatal stroke or myocardial infarction in hypertensive patients. Am J Hypertens. 2003;16:1011-7. doi:10.1016/j.amjhyper.2003. 07.018.

17. Knaapen AM, Ketelslegers HB, Gottschalk RW, Janssen RG, Paulussen AD, Smeets HJ, et al. Simultaneous genotyping of nine polymorphisms in xenobiotic-metabolizing enzymes by multiplex PCR amplification and single base extension. Clin Chem. 2004;50:1664-8. doi:10.1373/clinchem.2004.034058.

18. Mack MJ. Clinical trials versus registries in coronary revascularization: which are more relevant? Curr Opin Cardiol. 2007;22: $524-8$.

19. Klungel OH, Martens EP, Psaty BM, Grobbee DE, Sullivan SD, Stricker $\mathrm{BH}$, et al. Methods to assess intended effects of drug treatment in observational studies are reviewed. J Clin Epidemiol. 2004;57:1223-31. doi:10.1016/j.jclinepi.2004.03.011. 